NASA Technical Memorandum 103200

\title{
Neutron and Gamma Irradiation Effects on Power Semiconductor Switches
}

(NASA-TM-103200) NEUTRON AND GAMMA IRRAOIATION EFFECTS ON POWER SEMICONDUCTOR SWITCHES (NASA) $8 \mathrm{P}$
CSCL $09 A$
N90-25278

Unclas

0291543

G.E. Schwarze

Lewis Research Center.

Cleveland, Ohio

and

\section{A.J. Frasca}

Wittenberg University

Springfield, Ohio

Prepared for the

25th Intersociety Energy Conversion Engineering Conference cosponsored by the AIChE, ANS, SAE, ACS, AIAA, ASME, and IEEE Reno, Nevada, August 12-17, 1990 


\section{NEUTRON AND GAMMA IRRADIATION EFFECTS ON POWER SEMICONDUCTOR SWITCHES}

\author{
G.E. Schwarze \\ National Aeronautics and Space Administration \\ Lewis Research Center \\ Cleveland, Ohio 44135
}

\author{
A.J. Frasca \\ Wittenberg University \\ Springfield, Ohio 45501
}

\section{ABSTRACT}

The performance characteristics of high power semiconductor switches subjected to high levels of neutron fluence and gamma dose must be known by the designer of the power conditioning, control and transmission subsystem of space nuclear power systems. Location and the allowable shielding mass budget will determine the level of radiation tolerance required by the switches to meet performance and reliability requirements. Neutron and gamma ray interactions with semiconductor materials and how these interactions affect the electrical and switching characteristics of solid state power switches is discussed. The experimental measurement system and radiation facilities are described. Experimental data showing the effects of neutron and gamma irradiation on the performance characteristics are given for power-type NPN Bipolar Junction Transistors (BJTs), and MetalOxide-Semiconductor Field Effect Transistors (MOSFETS). BJTs show a rapid decrease in gain, blocking voltage, and storage time for neutron irradiation, and MOSFETs show a rapid decrease in the gate threshold voltage for gamma irradiation.

\section{INTRODUCTION}

The electrical components used in the electrical power and control subsystem of space nuclear power systems will be subjected to gamma rays and neutrons emanating from the nuclear reactor. The level of radiation tolerance required by these components will be determined by their location and the allotted radiation shielding. High power switching devices will be one of the critical electrical components needed to condition, control, and regulate the power to the spacecraft's bus and any required ballast loads.

Under the Power Management element [1] of the Civilian Space Technology Initiative-High Capacity Power Program, the NASA-Lewis Research Center has initiated a program to characterize and evaluate the effects of neutrons and gamma rays on the electrical and switching performance of commercial and developmental-type solid state power switches. The primary focus of this switch program is to experimentally determine both the types and rates of degradation in the switch's performance due to radiation damage. The results obtained under this program should enable the circuit designer to determine the useability of a particular switch for a specified circuit application. This paper will be limited to a discussion of radiation damage in power-type BJTs and MOSFETs and experimental results will be presented to show how radiation damage affects these devices.

The physics of radiation damage in solid state switches is quite complex. The brevity of this paper does not allow a complete discussion of all of the effects associated with radiation in electronic materlals and devices so the interested reader is referred to references [2] to [4] for a more in-depth exploration of the subject.

\section{GAMMA RAY INTERACTIONS}

Gamma rays are photons or quanta of high energy and can be produced when a nucleus decays from its excited state to another lower or ground state. Gamma rays interact with matter in three different ways: photoelectric effect, Compton scattering and pair production. Electrons are emitted in each of these processes. Charged particles such as electrons, protons, and alpha particle interact with atoms primarily by Rutherford scattering (Coulomb scattering) and cause both ionization and atomic displacements.

In the photoelectric effect the incident gamma ray is completely absorbed by a tightly bound atomic electron ( $K$ or $L$ shell) and the bound electron, called the photoelectron, is ejected from the atom. Compton scattering involves an elastic collision with a loosely bound or "free" electron. However, as the energy of the incident gamma ray increases, Compton scattering can take place with the bound electrons. In Compton scattering the gamma ray is scattered at a reduced energy and the remainder of the incident gamma ray's energy becomes the kinetic energy of the scattered electron. In pair production the gamma ray passes near a nucleus and converts into an electron-positron pair. A gamma ray energy of at least $1.02 \mathrm{MeV}$ is required for this process. In sllicon the photoelectric effect dominates at photon energies less than $50 \mathrm{keV}$ and pair production dominates at energies greater than $20 \mathrm{MeV}$ with Compton scattering dominating in the intervening energy range.

\section{NEUTRON INTERACTIONS}

Neutrons interactions with matter can be divided into two categories: capture and scattering. In the capture process the target nucleus absorbs the incident neutron to form a compound excited nucleus 
which subsequently decays to a stable nucleus through photon and charged particle emission. In certain heavy nuclei the compound nucleus splits into two separate fragments accompanied by the emission of neutrons and gamma rays.

In scattering interactions the incident neutron remains free at a lower kinetic energy and part of the incident neutron's kinetic energy is transferred to the target nucleus known as the primary recoil or knock-on atom. Scattering interactions consist of elastic and inelastic collisions. In elastic scattering the incident neutron collides with the target atom with the incident neutron's energy being reduced by the kinetic energy imparted to the recoil atom. In inelastic scattering the neutron is first captured by the target atom to form a compound nucleus from which a lower energy neutron is emitted and the excited nucleus decays by gamma emission.

In scattering interactions the target atom in crystalline materials can be displaced from its lattice site and for most materials about $25 \mathrm{eV}$ is required to dislodge the atom. If the displaced atom is unable to find a vacant lattice site, then vacancy-interstitial pairs are generated to form simple or point defects in the crystal. The kinetic energy of the recoil atom determines whether it has additional elastic collisions with other lattice atoms or whether it dissipates its energy by ionization. Defect clusters form when a primary recoil atom is imparted with sufficient kinetic energy to cause additional collisions and atomic displacements. Defect clusters are regions that contain large numbers of relatively closely spaced defects.

Neutron scattering can generate a cascade of displaced and ionized atoms and these radiationinduced defects can cause permanent damage that alters the physical and electrical properties of crystals. The dominant interaction in silicon for fast neutrons is the scattering process which primarily forms defect clusters.

\section{IONIZATION EFFECTS}

Ionization is the process of renoving orbital electrons from atoms and producing positive ions and free, or unbound electrons. In semiconductors and insulators, ionization always produces electron-hole pairs; an electron is removed and a mobile hole is generated in the valence band. Both gamma rays and neutrons cause ionization but gamma rays are the predominant cause. The number of electron-hole pairs generated depends on the material's band gap and the quantity of energy absorbed by the ionized electron; an ionized electron with sufficient kinetic energy can cause additional ionization. About $3.6 \mathrm{eV}$ is needed to create an electron-hole pair in silicon. In semiconductor devices the main effects of ionization are hole traps or positive charge buildup in the insulator or passivation layer, electronic or interface states at the surface between the insulator and semiconductor and photocurrents. In positive charge bulld-up some of the electrons from the electron-hole pairs leave the insulator so that the trapped holes produce a net positive charge in the insulator. Electrons localized at the insulator-semiconductor interface can generate interface energy states distributed throughout the band gap. These interface or surface states charge and discharge as a function of bias and thus, affect charge distribution within a device. Photocurrents are caused by electronhole pairs generated in the junction's depletion region and by minority carriers within a diffusion length of the depletion region.

\section{DISPLACEMENT EFFECTS}

Atomic displacement is the process of dislodging an atom from its lattice site. Both neutrons and ganma rays cause displacement; gamma rays, through ionized electrons, usually cause simple defects while neutrons, the predominant displacement mechanism, usually cause cluster defects. Through thermal motion some of these displaced atoms become mobile and migrate through the lattice structure until they either recombine as vacancy-interstitial pairs, or form immobile stable defects, or escape to a free surface. The thermal annealing process depends on temperature, time and charge state. The charge state is the energy level the hole or electron occupies in the band gap.

In crystalline substances these atomic displacements alter the periodicity of the lattice. In general, the disruption of lattice periodicity in a semiconductor or insulator leads to discrete energy levels in the material's band gap. The presence of these discrete energy levels in the band gap can cause several processes to occur: generation, recombination, trapping, removal and tunneling of electron and hole carriers. Anyone, a combination of, or all of these processes can occur through the same energy level of the band gap. The process that dominates is determined by such variables as carrier concentration, temperature, and the particular region of a semiconductor device such as the $p-n$ junction depletion region. Besides these processes, radiation-induced atomic displacements generate carrier scattering centers which affect carrier mobility.

Carrier trapping occurs at shallow energy levels in the band gap and the same carrier that is captured by a trap is later emitted with no recombination taking place. Carrier removal alters carrier concentration and occurs when the dopant is compensated to cause a reduction in the equilibrium majority carrier concentrations. Tunneling is the process where carriers "tunnel" through a potential barrter such as in a reverse-biased $p-n$ junction. Carrier action in a semiconductor includes drift, diffusion, and recombination-generation. Drift is the motion of charged particles in response to an electric field. Diffusion is a thermal process where particles migrate from high to low regions of concentrations such that uniform distribution is achleved. Recombination-Generation $(R-G)$ is a process that indirectly affects current flow by changing the carrier concentration involved in the drift and diffusion process.

Generation is the creation of electron-hole pairs while the reverse process, recombination, is the 
annihilation of electron-hole pairs. The recombination lifetime is a function of $R-G$ center density and is the mean-time a minority carrier exists before recombining with a majority carrier. Both direct and indirect thermal $R-G$ processes occur in semiconductors. Direct thermal $R-G$ processes occur without an intermediary while indirect R-G processes require a 'third party' and take place only at sites in the material called $R-G$ centers. R-G centers are caused by introducing spectal impurity atoms such as gold into the lattice or by lattice defects resulting from processing or radiation damage. $R-G$ centers introduce allowed energy levels or electronic states near the center of the band gap.

Under equilibrium conditions (no external voltages, magnetic fields or stresses applied) the $R-G$ rates are equal so there is no net change in carrier concentration. Perturbation of the semiconductor such as the application of an external voltage causes elther the recombination or generation rate to change in such a direction to favor restoration of equilibrium conditions. The recombination rate increases if the perturbation causes an increase in carriers while the generation rate increases if the perturbation causes a decrease in carriers. Thus, R-G action causes a change in carrier concentration when equilibrium conditions are disturbed.

\section{EXPERIMENTAL SETUP}

The research facilities and equipment of the NASALewis Research Center, Wittenberg University, Ohio State University (OSU) and the University of Cincinnati (UC) are used for the experimental tests and data analysis. The OSU research reactor facility is used for neutron irradiation and the UC Cobalt-60 facility is used for gamma irradiation. At present both the neutron and gamma irradiation tests are done under room temperature conditions but higher temperature tests are planned to investigate the effect of thermal annealing during irradiation. The room temperature test fixture is made from polyethylene and symmetrically holds up to four devices. The fixture's size is limited by the port hole size at the OSU reactor. Both power and sense cables are provided for each test device and these cables extend outside either the neutron or gamma source to the test instruments. The inner surface and the four individual test sockets of the fixture are lined with cadmium to absorb the thermal neutrons, leaving only the epicadmium flux $(0.5 \mathrm{eV}$ to $10 \mathrm{MeV})$. The reason for thermal neutron removal is to more clearly represent a fast spectrum reactor and to minimize neutron activation of the irradiated devices so that immediate postirradiation tests can be conducted outside the reactor.

A curve tracer is used to make the electrical measurements before, during, and after irradiation. These measurements consist of the ON-state currentvoltage characteristic curves and the OFF-state leakage current-voltage breakdown curves. Special drive circuits are designed for the switching time tests for each of the different solid state switches. Presently, the tests for determining turn-on and turn-off times are conducted only before and after irradiation.

\section{RADIATION EFFECTS IN BJTS}

The BJT is a current controlled device in which the base current $I_{B}$ controls the output collector current $I_{C}$. Both neutrons and gamma rays affect the electrical and switching properties of BJTs. Fast neutrons primarily cause cluster defects and are the dominant radiation-induced damage mechanism in BJTs. High doses of gamma rays are capable of generating simple defects by energetic electrons resulting primarily from Compton scattering. The number of atomic displacements caused by neutrons is very large relative to gamma rays.

The result of both simple and cluster defects in silicon is the formation of R-G centers. Electronhole recombination causes reductions in current gain and switching times. Electron-hole generation causes an increase in leakage current. Majority carrier removal and decreases in carrier mobility cause increases in forward collector-emitter voltage $V_{C E}$. Neutron fluences greater than 1013 $\mathrm{n} / \mathrm{cm}^{2}$ are required for significant reductions in majority carrier density and mobility.

The current gain hFE of a BJT is the ratio of $I_{C}$ to $I_{B}$. h he is determined by the fraction of majority carriers emitted by the emitter that pass through the base as minority carriers and collected as majority carriers by the collector. An increase in $R-G$ center density due to radiation-induced defects causes a decrease in minority carrier lifetime so that the rate of electron-hole recombination increases in the base. Thus, hFE decreases as the fraction of emitter-injected carriers reaching the collector decreases so that $I_{C}$ decreases for a constant $I_{B}$, or equivalently, $I_{B}$ must increase to maintain a constant $I_{C}$. A switching-type power $B J T$ has a relatively low $h_{F E}$ at rated $I_{C}$ and $V_{C E}$ so any degradation in h $h_{F E}$ impacts the switch's operational use. A gain loss does not make a switching-type power BJT inoperative as long as the device does not exceed its maximum continuous $I_{B}$ rating, but gain loss does make it an inefficient switch because the switch's total losses increase as $I_{B}$ increases.

Figure 1 shows the rapid decrease in $h_{\mathrm{FE}}$ for increasing neutron fluence for three values of $I_{C}$. The test device was an NPN D60T455010 power transistor and is rated by the manufacturer for an $h_{F E}=10$ at $I_{C}=50 \mathrm{~A}$ and $V_{C E}=2.5 \mathrm{~V}$ and $a$ maximum $I_{B}=20 \mathrm{~A}$. The $I_{C}=10 \mathrm{~A}$ and $30 \mathrm{~A}$ curves are for a total fluence of $1.6 \times 10^{13} \mathrm{n} / \mathrm{cm}^{2}$. The $I_{G}=50 \mathrm{~A}$ curve terminates at a fluence of $0.74 \times 10^{13} \mathrm{n} / \mathrm{cm}^{2}$ because above this fluence $I_{B}$ exceeds $20 \mathrm{~A}$. Extrapolation of the $I_{C}=50$ A curve shows that as the fluence approaches $1013 \mathrm{n} / \mathrm{cm}^{2}$, h $\mathrm{FE}$ approaches unity. All three curves show quite clearly that density of $R-G$ centers which cause a rapid degradation in hFE. The $37 \mathrm{krad}$ gamma dose is due to fission fragment decay and has no significant effect on the gain degradation. On gain considerations alone, the operational usefulness of power BJTs tend to be limited to fluences less than $1012 \mathrm{n} / \mathrm{cm}^{2}$. 
In a reverse-biased $p-n$ junction the drift current dominates and this reverse current, usually called the leakage current, consists of two components. The first component is the reverse saturation current so named because it is almost independent of the reverse-bias voltage after a few tenths of volts. This component assumes a zero $R-G$ rate in the depletion region and the magnitude of this current is inversely proportional to the minority carrier diffusion length and is thus 1 imited by the supply of thermally generated minority carriers. The diffusion length is proportional to the square root of the minority carrier lifetime so the saturated reverse current increases as the minority carrier iffetime decreases. The second component of the leakage current assumes $R-G$ does occur in the depletion region. Under reverse-bias the carrier concentrations in the depletion region are less than their thermal equilibrium values. The electron-hole generation rate must thus increase to restore the carrier concentrations to their thermal equilibrium values. This leakage current component is directly proportional to the thermal generation rate which is inversely proportional to the recombination lifetimes of the electrons and holes. As the R-G center density increases due to radiation-induced atomic displacements, the thermal generation rate increases so that this current component increases.

Since both leakage current components increase for decreasing minority carrier lifetime, it follows that the total leakage current increases as the density of radiation-induced defects increase. Thus, for a specified reverse collector-base voltage with open emitter, $V_{C B O}$ and specified collector-emitter voltage with open base, $V_{C E O}$, the respective leakage currents $I_{C B O}$ and I $I_{C E}$ increase as the radiation-induced defects increase, or equivalently, if $I_{C B O}$ and $I_{C E O}$ are specified, then the respective blocking voltages $V_{C B O}$ and $V_{C E O}$ will decrease as the number of defects increase.

Figure 2 is a plot of $V_{C E O}$ for $I_{C E O}=10 \mu \mathrm{A}$ as a function of neutron fluence for four devices. Again the test device is the D60T455010 and the manufacturer's rating for $V_{C B O}$ and $V_{C E O}$ are $500 \mathrm{~V}$ and 450 respectively at $25 \mathrm{C}$. Two of the devices were tested at an epicadmium neutron flux of $7,55 \times$ $10^{6} \mathrm{n} / \mathrm{cm}^{2} \mathrm{~s}$ for a fluence of $1.65 \times 10^{6} \mathrm{n} / \mathrm{cm}^{2}$ and total gamma dose of 37 krads. The other two devices were tested at half this flux with corresponding reductions of a factor of two in fluence and gamma dose. The devices with initially high values of $V_{C E O}$ have faster degradation rates but in all cases, $\nabla_{C E O}$ progressively decreases with increasing fluence. Thus, as fluence increases to cause increased atomic displacements, the thermal generation rate of carriers must increase because minority carrier recombination lifetime decreases and so $V_{C E g}$ must continue to decrease for a fixed $I_{C F O}$. It should be noted that the $V_{C B O}$ curves as a function of fluence for these same devices show similar rates of degradation. From operational considerations the use of power BJTs for neutron fiuences approaching $10^{13} \mathrm{~W}^{\mathrm{cm} \mathrm{m}^{2}}$ becomes quite device's forward voltage blocking capability.
The four states in the switching sequence of a BJT are OFF, turn-on, ON, and turn-off. The OFF-state, the non-conduction interval, is characterized by $V_{C B O}$ and $V_{C F O}$ and their respective leakage currents $I_{C B O}$ and ICEO. The ON-state, the conduction interval, is characterized by $h_{F E}$ at specified $I_{C}$ and $V_{C E}$ values. The turn-on time is the sum of the delay rise and rise time and depends on how fast the majority carriers injected into the base by the emitter, can be collected by the collector for a specified output current. During turn-on, excess minority carriers accumulate in the base as the BJT goes from cut-off through the active to the saturation region. Excess minority carrier charge accumulated during turn-on must all be removed during turn-off. The deeper the BJT is driven into saturation, the longer the rise time and the larger the amount of excess charge accumulated in the base. The turn-off time is the sum of the storage and fall times and depends on how fast the excess minority carriers recombine. For a BJT operating in the saturation region, the storage time is the longest switching time interval compared to the other three intervals and so becomes the most important time in determining the switching speed.

The excess carrier charge accumulated during turnon and the rate of recombination of this excess charge during turn-off is proportional to the minority carrier lifetime in the base. Faster switching times mean lower switching losses and one means to reduce storage time is to purposely introduce $R-G$ centers with electronic states near the center of the band gap. The use of gold as an impurity is a common method to increase switching speeds by decreasing minority carrier lifetime. Since radiation-induced atomic displacements introduce $R-G$ centers near midgap, it becomes evident that these radiation-induced defects will reduce storage time.

Figure 3 shows the pre- and post-irradiation storage time for the D60T455010 as a function of $I_{B}$ for $I_{C}-40 A$ and collector supply voltage $V_{C C}=45$ $v$. Both devices were subjected to a neutron flux of $7,55 \times 10^{6} \mathrm{n} / \mathrm{cm}^{2} \mathrm{~s}$ for a fluence of $1.65 \times 10^{13}$ $\mathrm{n} / \mathrm{cm}^{2}$ and background gamma dose of $37 \mathrm{krads}$. The curves clearly show a significant reduction of almost $100 \%$ in storage time due to the radiationinduced de-fects. The decrease in minority carrier lifetime caused by the neutron-induced atomic displacements clearly enhances the switching characteristics of BUTs but likewise, severely degrades the ON-state current gain and the OFFstate forward blocking voltage capability.

\section{RADIATION EFFECTS IN MOSFETS}

The MOSFET is a voltage controlled device in which the gate-source voltage controls the output drain current. MOSFETs are of two types: depletion and enhancement-mode. Both types can be either $\mathrm{N}$ - or $P$ - channel devices. Depletion-mode MOSFETs turn on with zero gate-source voltage and are normally-on devices so a gate drive is required for turn-off and to maintain the blocking voltage in the OFFstate. Enhancement-mode MOSFETs are normally-off devices because a gate-source threshold voltage 
VGS(th), is required for turn-on. Turn-on is accomplished by developing a charge inversion layer in the semiconductor under the gate oxide to form the conducting channel. Both $\mathrm{N}$-and P-channel enhancement-mode MOSFETs have been developed for power applications with $\mathbf{N}$-channel devices having the largest selection of current and voltage ratings. Accordingly, $\mathrm{N}$-channel enhancement-mode MOSFETs will be the basis for the discussion of radiation-induced effects.Unlike BJTs, MOSFETs conduct current by majority carriers only and the effects of radiation damage in MOSFETs is different from those in BJTs. Ionizing radiation, primarily due to gamma rays causes $V_{G S}(t h)$ shifts, and atomic displacements, primarily due to neutrons, causes increases in ON-state resistance $\mathrm{R}_{\mathrm{DS}}(\mathrm{th}) \cdot$

Ionizing radiation generates electron-hole pairs almost uniformly in the gate oxide. The number of pairs generated is dependent on oxide thickness, band gap of the oxide, and energy absorbed. The electrons and holes are separated by an applied or radiation-induced electric field in the oxide. Some of the electrons will recombine immediately with the holes and the remainder will move quickly to the metal gate terminal. The remaining holes drift slowly toward the oxide-semiconductor interface. Some of the holes pass into the semiconductor and others are captured in traps in the oxide near the interface to cause positive charge build-up which is dependent on temperature, density of traps available at the interface, oxide thickness, oxide processing techniques and magnitude and direction of the electric field. The trapped holes act like a positive gate bias and so they cause charge inversion in the semiconductor near the interface. The positive charge build-up causes a negative shift in $V_{G S}(t h)$ so that a less positive gate bias is required for turn-on. Sufficient positive charge build-up can cause $V_{G S}$ (th) to be equal to or less than zero. For $V$ GS $\leq 0$ the device becomes like a depletionmode MOSFET and turns on without any gate bias and indeed, a negative bias is required for turn-off and maintenance of the OFF-state.

Imperfections and irregularities at the surface of a crystal cause disruptions in lattice periodicity and introduce energy levels in the semiconductor band gap. Since surface or interface effects give rise to these energy states they are called interface or surface states and the probability that electrons will occupy these states depends on the state's position in the band gap. This position is strongly dependent on the surface potential due to applied bias and positive charge build-up. Radiation-induced ionization can change both the status and density of these interface states. The formation of negatively charged interface states can partially compensate for the positive charge build-up in the oxide by repelling electrons from the channel near the semiconductor-oxide interface. As the gamma dose increases, this compensation effect can increase to cause a decrease in the rate of negative shift in $V_{G S(t h)}$ to the point that $V_{G S(t h)}$ moves in a positivedirection.

The effects of Co-60 gamma rays on $V_{G S}$ (th) is shown in Figure 4 for two N-channel MTMISN5O entancement- mode power MOSFETs. The manufacturer rates this device for a drain-source voltage $V_{D S}=500 \mathrm{~V}$, drain current $I_{D}=15 \mathrm{~A}$, and maximum $V_{G S}$ (th) $=4.5 V$ at 25 C. The test devices were subjected to a 6.8 $\mathrm{krad} / \mathrm{hr}$ garma dose rate for $73 \mathrm{krads}$ of dose. No bias voltages were applied during irradiation except for purposes of data collection. The curve in Figure 4 shows that both devices track each other well and that $V_{G S}(t h)$ decreases moderately up to about 5 krads, after which $V_{G S}(t h)$ decreases rapidly. The slope of the curve suggests that $V_{G S}(t)$ will act like a depletion-mode MOSFET above 180 krads unless interface states begin to develop rapidly to compensate positive charge build-up.

Radiation-induced crystal defects caused by atomic displacements cause decreases in majority carrier density (carrier removal) and carrier mobility. The electrical conductivity is proportional to the product of majority carrier concentration and mobility so a reduction in channel conductivity causes RoS(on) to increase. Majority carrier mobility can also decrease due to scattering caused by the positive charge build-up and interface states, but these affect RDS(on) only at very high ionizing doses. The neutron-induced displacements are the dominant mechanism for causing increases in RoS(on).

Figure 5 shows the effect of neutrons on $R_{D S}$ (on for two MTMI5N50 MOSFETs. The two test devices were subjected to neutron fluxes from $7.55 \times 10^{7}$ to $2.26 \times 10^{9} \mathrm{n} / \mathrm{cm}^{2} \mathrm{~s}$ for a total fluence of $3.8 \times 10^{13}$ $\mathrm{n} / \mathrm{cm}^{2}$ and a background gamma dose of $70 \mathrm{krads}$. Again, no bias voltages were applied during irradiation except during data collection. Both devices show no significant increase in RDS(on) for fluences up to $10^{13} \mathrm{n} / \mathrm{cm}^{2}$ after which $\mathrm{R}_{D S}$ (on) begins to increase. In terms of radiation hardness the experimental resuits indicate that this high voltage MOSFET is afceptable for neutron fluences up to at least $10^{13} \mathrm{n} / \mathrm{cm}^{2}$ and possibly $1014 \mathrm{n} / \mathrm{cm}^{2}$ but approaches unacceptability as an enhancement-mode device for gamma doses beyond 50 krads. So called "rad hard" enhancement-mode power MOSFETs are now becoming available that show considerable improvement in their resistance to tonizing radiation. Several manufacturers [5,6] advertise the availability of rad-hard devices up to 1 Megarad.

\section{ACKNOWLEDGMENT}

This research was sponsored by the NASA Lewis Research Center under the High Capacity Power Element of the Civilian Space Technology Initiative.

\section{REFERENCES}

1. Schwarze, G. E., "Overview of Space Power Electronics Technology Under the CSTI High Capacity Power Program", Proceedings Seventh Symposium on Space Nuclear Power Systems. Conf. 900109, Alburquerque, Hew Mexico, January $7-10,1990$. 
2. Gover, J. E. and Srour, J. R., "Basic Radiation Effects in Nuclear Power Electronics Technology" Sandia Report SAND 85-0776, May 1985.

3. Srour, J. R., et al, Radiation Effects on and Dose Enhancement of Electronic Materials, Noyes Publications, New Jersey, 1984.

4. van Lint, V. A. J., et al, Mechanisms of Radiation Effects in Electronic Materials. Volume 1, John Wiley \& Sons, New York, 1980.

5. Wheatley, C. F., "Radiation Hardening of Vertical DMOS (VDMOS) Power MOSFETs", G. E. Power MOSFETs Application Note AN-8831, December 1988.

6. "HEXFET POwer MOSFET Designer's Manual " HDB-4, Fourth Edition, International Rectifier, El Segundo, California, 1987, pp G-727 to G761.
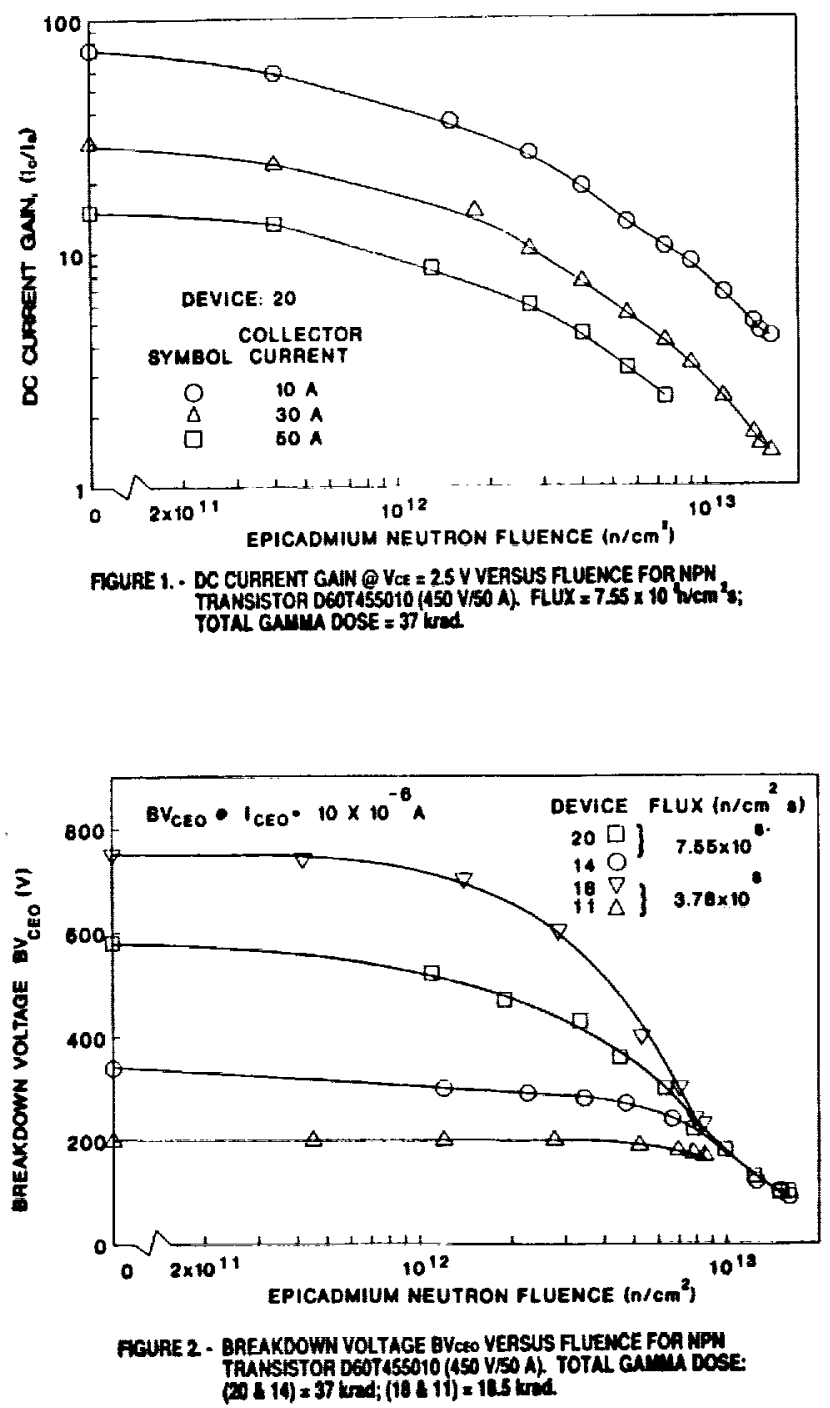
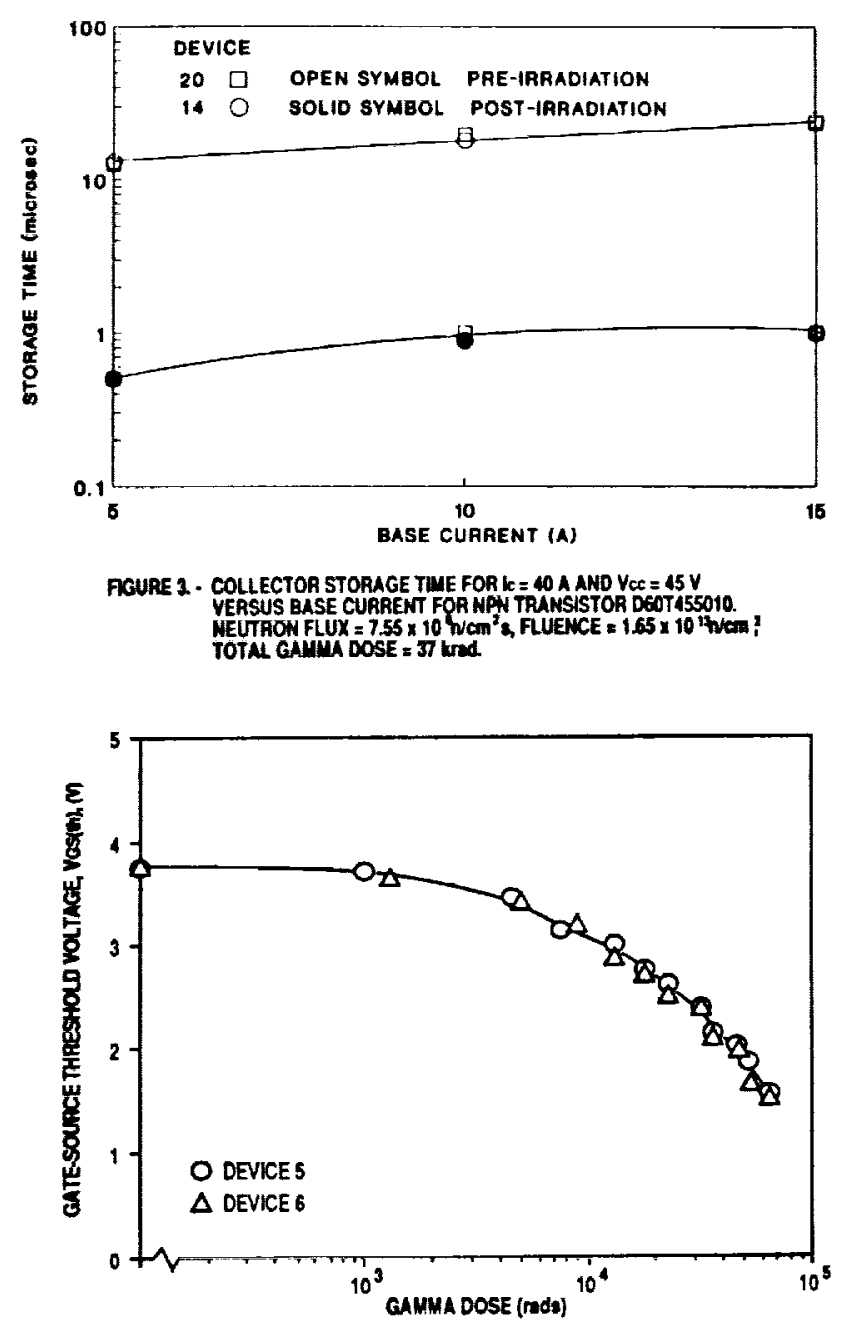

FIGUAE 4. GATE.SOURCE THRESHOLD VOLTAGE YEASUS GAMMA DOSE FOR W CHANNEL ENHANCEMENT.MODE MOSFET WTM 1SN30 (SOOVASA), GAMMA DOSE RATE $=6.8 \mathrm{krds} / \mathrm{hr}$

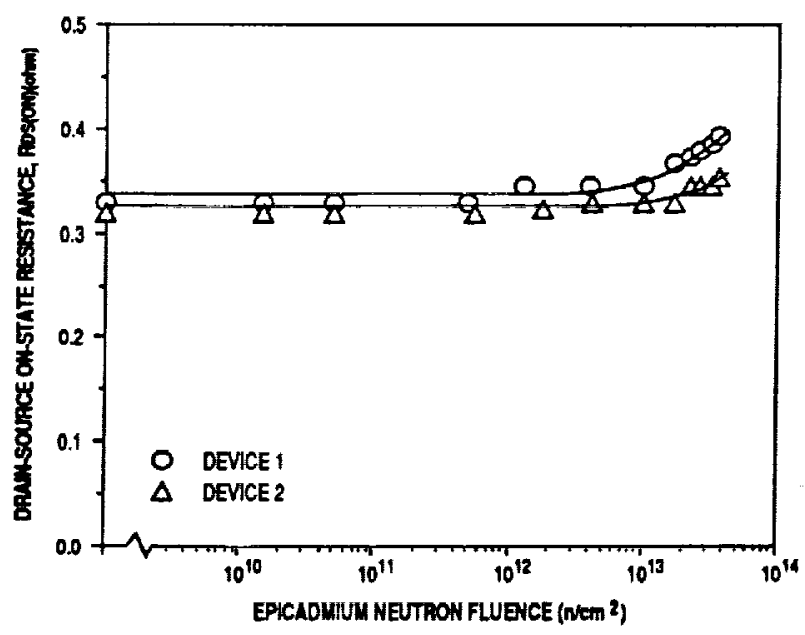

FIGURE 5. OHAIN-SOUACE ON-STATE RESISTANCE FOR $10=1504$ AND VOS(n) $=8.5 \mathrm{~V}$ VERSUS EPICADMIUM NEUTAON FLUENCE FOR N CHANNEL WTM ISN50 (50OV/13A). NEUTRON FLUX $=7.55 \times 107$ TO $2.26 \times 10^{\circ}$ nem2 GALMA bOSE $=70$ kreds. 


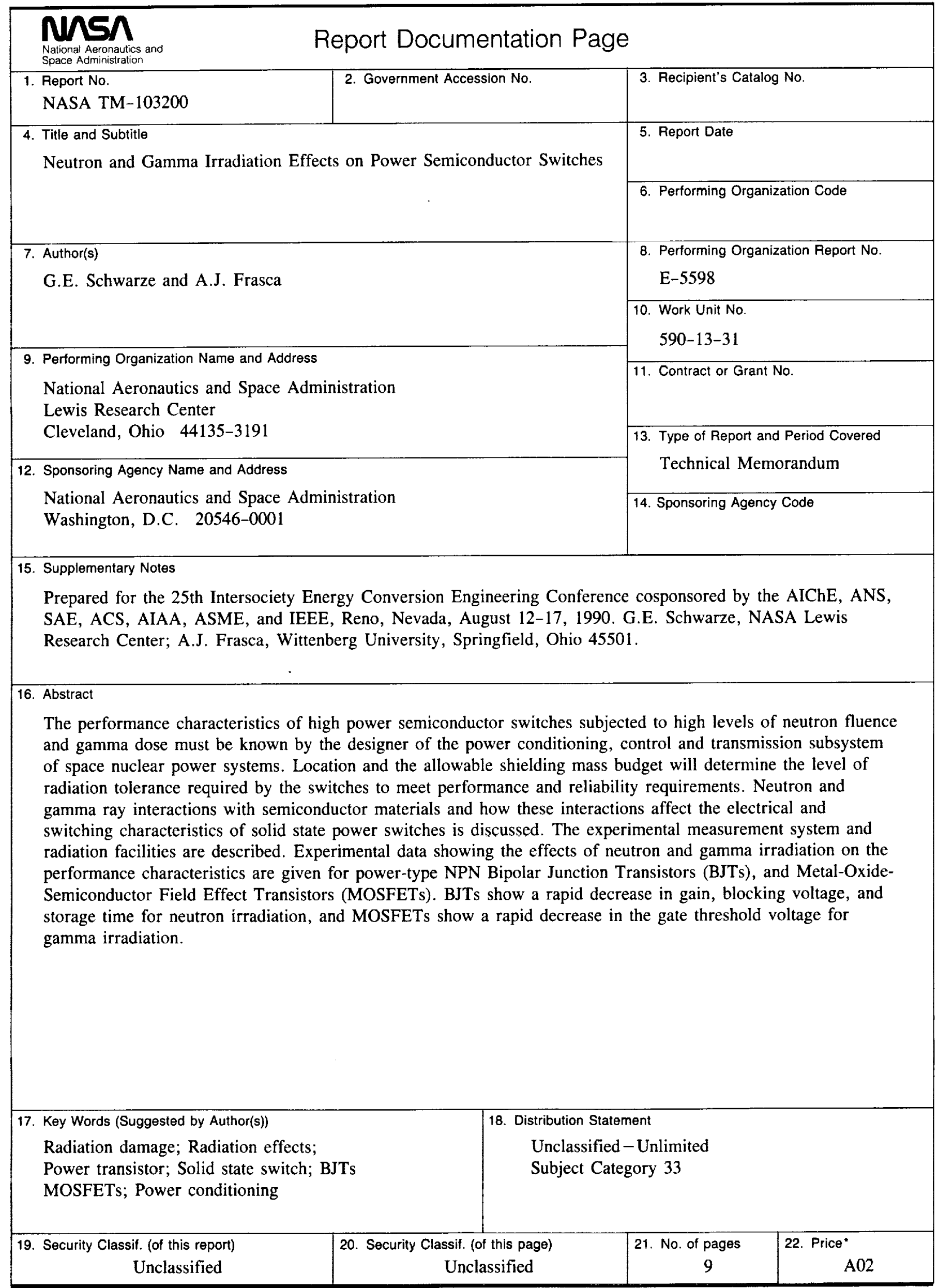


